\title{
G6PD deficiency in Hansen's disease: A preventable peril!
}

\author{
Arnab Ghorui ${ }^{1}$ and Pratap Patra ${ }^{1}$ \\ ${ }^{1}$ All India Institute of Medical Sciences - Patna
}

January 26, 2022

\begin{abstract}
Although under-reported, hemolytic anemia is common with dapsone therapy. However, it is prudent to screen underlying G6PD deficiency to prevent potentially life-threatening episode of intravascular hemolysis in leprosy.
\end{abstract}

G6PD deficiency in Hansen's disease: A preventable peril!

Dr. Arnab Kumar Ghorui, MBBS

Department of Pediatrics

All India Institute of Medical Sciences, Patna

Dr. Pratap Kumar Patra, MD,DM.

Department of Pediatrics

All India Institute of Medical Sciences, Patna

Correspondence to:

Dr. Pratap Kumar Patra, MD,DM.

Associate Professor

Department of Pediatrics

All India Institute of Medical Sciences

Patna, Bihar, India.

Ph:91-7766932165

Consent: Written consent has been obtained from the parents to publish this image.

G6PD deficiency in Hansen's disease: A preventable peril!

An 11-year-old boy noted to have a hypopigmented spot over left cheek with diminished sensation (Figure 1A). Thickening of bilateral ulnar nerves were additional findings. Hansen's disease was diagnosed, and he was treated with clofazimine, dapsone, and rifampicin locally. After three days he developed fever, yellow discoloration of eyes, and cola-colored urine (Figure 1B). On examination, severe pallor, icterus, and splenomegaly was noted. Investigation revealed hemoglobin $58 \mathrm{~g} / \mathrm{L}$, indirect bilirubin $5.3 \mathrm{mg} / \mathrm{dL}$, serum lactate dehydrogenase $5619 \mathrm{U} / \mathrm{L}$ (Normal $<170 \mathrm{U} / \mathrm{L}$ ), aspartate aminotransferase $210.9 \mathrm{U} / \mathrm{L}$ (Normal $<40$ $\mathrm{U} / \mathrm{L}$ ). Level of G6PD was significantly reduced. He required two units packed red blood cell transfusion along with 1.5 times maintenance fluid and injection lasix. He recovered after seven days of hospitalization. 
Although under-reported, hemolytic anemia is common and related to N-hydroxy metabolite of dapsone (1). However, concomitant G6PD deficiency enhances the risk of intravascular hemolysis, which may be severe, and a cause of significant morbidity. Furthermore as the prevalence of G6-PD deficiency in India varies from $2.3 \%$ to $27 \%$, there may be a chance that both conditions exists simultaneously (2). Therefore, before prescribing dapsone containing regimen in leprosy, it is prudent to screen underlying G6PD deficiency in boys to avoid a catastrophic episode of intravascular hemolysis.

Key words: Hansen's disease, Dapsone, G6PD deficiency

Authors contribution: Arnab Ghorui : patient managementdata collection, manuscript writing, and editing.

Pratap Kumar Patra : Patient management, manuscript writing, and final approval.

\section{Authors contribution:}

Figure legends:

Figure 1 A: Hypopigmented lesion over the left cheek

Figure 1 B: Image of cola colored urine

\section{References:}

1. Deps P, Guerra P, Nasser S, Simon M. Hemolytic anemia in patients receiving daily dapsone for the treatment of leprosy. Lepr Rev. 2012 Sep;83(3):305-7.

2. Mukherjee MB, Colah RB, Martin S, Ghosh K. Glucose-6-phosphate dehydrogenase (G6PD) deficiency among tribal populations of India - Country scenario. Indian J Med Res. 2015 May;141(5):516-20.

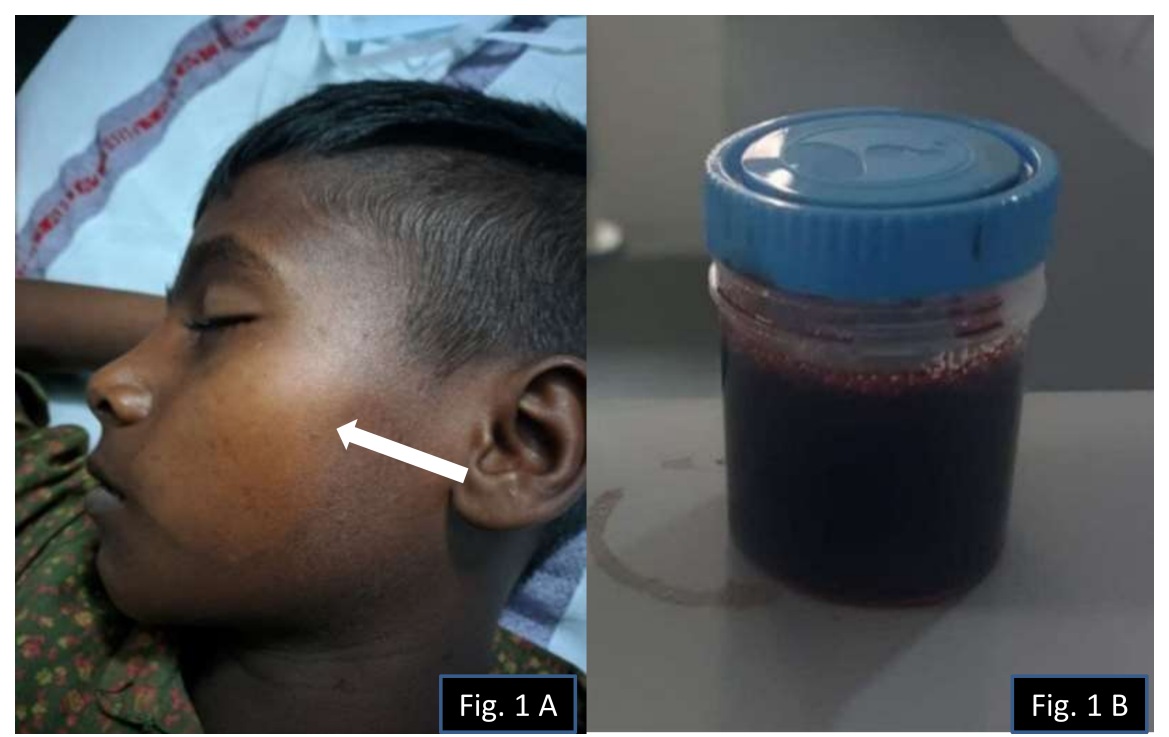

